\title{
The interstellar cosmic-ray electron spectrum from synchrotron radiation and direct measurements ${ }^{\star}$
}

\author{
A. W. Strong ${ }^{1}$, E. Orlando ${ }^{2,1}$, and T. R. Jaffe ${ }^{3,4}$ \\ ${ }^{1}$ Max-Planck-Institut für extraterrestrische Physik, Postfach 1312, 85741 Garching, Germany \\ e-mail: aws@mpe.mpg.de \\ 2 W.W. Hansen Experimental Physics Laboratory, Kavli Institute for Particle Astrophysics and Cosmology, Stanford University, \\ Stanford, CA 94305, USA \\ e-mail: eorlando@stanford.edu \\ 3 Université de Toulouse; UPS-OMP, IRAP, Toulouse, France \\ ${ }^{4}$ CNRS, IRAP, 9 Av. colonel Roche, BP 44346, 31028 Toulouse Cedex 4, France
}

Received 4 March 2011 / Accepted 17 August 2011

\begin{abstract}
Aims. We exploit synchrotron radiation to constrain the low-energy interstellar electron spectrum, using various radio surveys and connecting with electron data from Fermi-LAT and other experiments.

Methods. The GALPROP programme for cosmic-ray propagation, gamma-ray and synchrotron radiation is used. Secondary electrons and positrons are included. Propagation models based on cosmic-ray and gamma-ray data are tested against synchrotron data from $22 \mathrm{MHz}$ to $94 \mathrm{GHz}$.

Results. The synchrotron data confirm the need for a low-energy break in the cosmic-ray electron injection spectrum. The interstellar spectrum below a few $\mathrm{GeV}$ has to be lower than standard models predict, and this suggests less solar modulation than usually assumed. Reacceleration models are more difficult to reconcile with the synchrotron constraints. We show that secondary leptons are important for the interpretation of synchrotron emission. We also consider a cosmic-ray propagation origin for the low-energy break.

Conclusions. Exploiting the complementary information on cosmic rays and synchrotron gives unique and essential constraints on electrons, and has implications for gamma rays. This connection is especially relevant now in view of the ongoing Planck and Fermi missions.
\end{abstract}

Key words. radio continuum: galaxies - ISM: magnetic fields - gamma rays: ISM - Galaxy: structure - cosmic rays

\section{Introduction}

Direct measurements of cosmic-ray (CR) electrons extend from $\mathrm{TeV}$ down to $1 \mathrm{GeV}$ (and lower energies from spacecraft like Ulysses and Voyager), but solar modulation complicates their intepretation at energies below about $10 \mathrm{GeV}$. Fermi-LAT (Abdo et al. 2009b; Ackermann et al. 2010) has made the currently most precise electron measurements in the range $7 \mathrm{GeV}-1 \mathrm{TeV}$; here modulation is of less importance although still significant in the lower part of this range. Synchrotron radiation (from tens of $\mathrm{MHz}$ to tens of $\mathrm{GHz}$ ) probes interstellar electrons from 0.5 to $20 \mathrm{GeV}$ for the typical Galactic magnetic field (hereafter B-field) of a few $\mu \mathrm{G}$, and hence can be used in conjunction with direct measurements to construct the full spectrum from $\mathrm{GeV}$ to $\mathrm{TeV}$. At low energies this will finally allow an independent estimate of solar modulation for testing heliopheric propagation models. In contrast such a probe of the low-energy interstellar spectrum is not available for $\mathrm{CR}$ nuclei.

An extensive review of CR propagation including electrons can be found in Strong et al. (2007); a recent global viewpoint for the Milky Way is given in Strong et al. (2010), and a propagation parameter study in Trotta et al. (2011). Further discussion of the electron spectrum measured by Fermi-LAT can be found

* The GALPROP parameter files is only available at the CDS via anonymous ftp to cdsarc.u-strasbg. fr $(130.79 .128 .5)$ or via http://cdsarc.u-strasbg.fr/viz-bin/qcat?J/A+A/534/A54 in Delahaye et al. (2010); Grasso et al. (2011); di Bernardo et al. (2011). Its relation to the high-energy positron excess discoved by the PAMELA experiment (Adriani et al. 2009) is also discussed in these papers. Our preliminary study of the synchrotron spectrum using GALPROP was given in Orlando et al. (2009).

The main objective of the present paper is to constrain the interstellar electron spectrum using a combination of the latest electron spectrum measurements and synchrotron radiation, leaving the question of its origin via injection and propagation as a side-issue. Hence while we use the CR propagation code GALPROP to generate interstellar spectra for various propagation scenarios, the latter are not our main focus. Nevertheless it is shown that some current models are actually excluded on the basis of the synchrotron data. The relation between synchrotron and electrons is complicated by the presence of secondary electrons and positrons, and hence these are included in our model and their effect is addressed.

In previous analyses we were able to constrain the total B-field $B_{\text {tot }}$ on the basis of synchrotron data and the distribution of cosmic-ray electrons derived from gamma rays (Strong et al. 2004a); we obtained a local value $B_{\text {tot }}=6 \mu \mathrm{G}$ and a scale length of $8 \mathrm{kpc}$ in $R$ and $1 \mathrm{kpc}$ in $z$. The value of $B_{\text {tot }}$ has frequently been quoted in the literature as an independent measurement of the total B-field. Since then the CR source distribution has been modified to better reflect the distribution of SNR as traced by pulsars (Strong et al. 2004b), and this influences the derived radial variation of $B_{\text {tot }}$; the larger radial variation 
of the source function implies a smaller variation of $B_{\text {tot }}$. Also the new measurement of the electron spectrum by Fermi-LAT (Abdo et al. 2009b; Ackermann et al. 2010) and the elimination by Fermi-LAT of the EGRET gamma-ray "GeV excess" (Abdo et al. 2009a) ${ }^{1}$ lead to an upward revision of the value of $B_{\text {tot }}$. We concentrate on the spectral aspects of synchrotron emission in this paper.

The magnitude of the B-field is a free parameter in this analysis, since while the regular component can be determined from rotation measures of pulsars and extragalactic sources, this is only a fraction of the total field. Our approach is to use the models for regular component derived from RMs, combining these with a random field to be determined, and the latter is one of the results of our analysis.

Extensions to lower frequencies where absorption is important have been made, see for example Strong \& Wolfendale (1978) and Webber \& Higbie (2008).

In a paper complementary to this one, we also address the relation between cosmic-ray electrons, synchrotron and B-fields ${ }^{2}$.

\section{GALPROP development}

A description of the GALPROP ${ }^{3}$ model can be found in Strong et al. (2007) and references therein; in particular see Strong \& Moskalenko (1998), Strong et al. (2004a) and Porter et al. (2008), and the GALPROP Explanatory Supplement available from the GALPROP website.

A shortcoming of GALPROP up to now has been the simplified B-field modelling, using only a random component and a simple 2D exponential dependence. With the availability of excellent radio continuum surveys from tens of $\mathrm{MHz}$ to tens of $\mathrm{GHz}$, including the WMAP satellite data, more sophistication is desirable; we have therefore introduced full 3D models for both regular and random B-field. For the present spectral study only the total field is required (derived from the 3D model), and a $2 \mathrm{D}$ propagation scheme is sufficient.

\subsection{Synchrotron emissivity calculations}

From the spectrum of particles (here electrons or positrons) computed by GALPROP at all points on the 3D grid, we integrate over particle energy to get the synchrotron emissivity for the regular and random fields.

The emissivity as seen by an observer at the solar position is computed as a function of $(x, y, z, v)$. The spectrum and distribution of the emissivity thus depends on the form of the regular and random components of the magnetic field, and the spectrum and distribution of CR leptons.

\footnotetext{
${ }^{1}$ Our earlier work was based on an electron spectrum adjusted to fit this excess, and which was a factor 4 higher than locally measured, while now we use the measured electron spectrum.

2 Jaffe et al. (2011). Both papers use the GALPROP code to model $\mathrm{CR}$ propagation. The main difference is that they consider emission in the Galactic plane from $408 \mathrm{MHz}$ to $23 \mathrm{GHz}$, while we use data out of the plane down to $22 \mathrm{MHz}$. They address the spatial variations induced by the B-field, while we concentrate only on the spectral aspects. We explicitly compare the contributions from secondary leptons, while they consider only the total. They use polarization (with Faraday rotation) to separate the regular and random B-field components. They also use rotation measures to constrain the regular B-field. Despite the differences in approach, the conclusions - on issues common to the papers - are consistent. A recent paper describing a similar approach to ours (Bringmann et al. 2011) has been brought to our attention.

3 The code is publicly available at http://galprop.stanford.edu
}

\subsubsection{Regular field}

The synchrotron emissivity (in erg $\mathrm{s}^{-1} \mathrm{~Hz}^{-1}$ ) of an isotropic distribution of monoenergetic relativistic particles in a uniform magnetic field has polarized components parallel and perpendicular to the projection of the field on the line-of-sight to the observer (Longair 2010):

$$
\begin{aligned}
& \epsilon_{\mathrm{par}}(v)=\frac{\sqrt{3}}{2} \frac{e^{3}}{m c^{2}} B_{\text {perp }}[F(x)-G(x)] \\
& \epsilon_{\mathrm{perp}}(v)=\frac{\sqrt{3}}{2} \frac{e^{3}}{m c^{2}} B_{\text {perp }}[F(x)+G(x)]
\end{aligned}
$$

where $x=v / v_{\mathrm{c}}$, with $v_{\mathrm{c}}=\frac{3}{4 \pi} \frac{e}{m c} B_{\text {perp }} \gamma^{2}$ and with $\gamma$ the electron Lorentz factor, $B_{\text {perp }}=B(x, y, z) \sin \alpha$, with $\alpha$ the angle between the magnetic field and the line-of-sight. The functions $F(x)$ and $G(x)$ are defined in terms of Bessel functions (Longair 2010), with:

$$
\begin{aligned}
& F(x)=x \int_{x}^{\text {inf }} K_{5 / 3}\left(x^{\prime}\right) \mathrm{d} x^{\prime} \\
& G(x)=x K_{2 / 3}(x)
\end{aligned}
$$

where $K_{5 / 3}(x)$ and $K_{2 / 3}(x)$ are the modified Bessel functions of order $5 / 3$ and $2 / 3$. They are conveniently provided as $\mathrm{C} \mathrm{li-}$ brary functions in the GNU Scientific Library ${ }^{4}$. The resulting synchrotron spectrum has a broad maximum centred roughly at the frequency $v_{\mathrm{c}}$ and the maximum has a value $v_{\max }=0.29 v_{\mathrm{c}}$ (Longair 2010).

The polarization formulation will be of use for our future work. Here we are only interested in the total intensity given by the sum of the two components described above:

$\epsilon(v, \gamma)=\sqrt{3} \frac{e^{3}}{m c^{2}} B_{\text {perp }} F(x)$

\subsubsection{Random field}

For a randomly oriented field the emissivity is isotropic and obtained by integrating the regular field expressions over all solid angles. The result is given by (Ghisellini et al. 1988)

$\epsilon_{\text {rand }}(v)=C x^{2}\left[K_{4 / 3} K_{1 / 3}-\frac{3}{5} x\left(K_{4 / 3} K_{4 / 3}-K_{1 / 3} K_{1 / 3}\right)\right]$

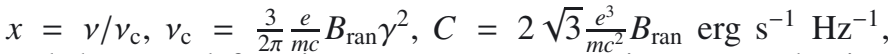
and the Bessel functions $K_{4 / 3}, K_{1 / 3}$ are again computed using the GNU Scientific Library.

Our implementation has been checked by integrating the regular field expression over solid angle, giving exact agreement with this formula.

\subsection{Synchrotron intensity}

With GALPROP calculation of emissivity on the grid, we integrate over the line-of-sight to get the synchrotron intensity for the regular and random fields. The synchrotron intensity at frequency $v$ is then given by

$I(v)=\int \epsilon(v) \mathrm{d} s$.

${ }^{4}$ http://www.gnu.org/software/gsl 
The observed brightness temperature ${ }^{5}$ of the radiation seen in a given direction is

$T(v) \propto \frac{c^{2} I(v)}{2 v^{2}}$.

The resulting synchrotron skymaps for a user-defined grid of frequencies are output by GALPROP in Galactic coordinates either as CAR (Carré projection) or in HEALPix (Górski et al. 2005) (the preferred format). The emissivity as seen by an observer at the solar position is also output as a function of $(R, z, v)$ (spatial 2D) or $(x, y, z, v)$ (spatial 3D).

\subsection{Galactic magnetic field models}

In the same way as for the other Galactic constituents (gas, ISRF, cosmic rays), the magnetic field is defined on a grid, in 2D or 3D. Only the 3D case is relevant for the full B-field model, although the $2 \mathrm{D}$ case is retained for compatibility.

In Strong et al. (2000) only the random component of the magnetic field was present and was implemented in 2D with an exponential law for the component.

Since that work, many 3D models of the Galactic magnetic field have been implemented in GALPROP in order to calculate the synchrotron emission from the Galaxy. The regular B-field used in the present work is the model RING-ASS of Sun et al. (2008) for the disk, based on rotation measures of extragalactic radio sources. This has typically $B_{\text {reg }}=2 \mu \mathrm{G}$. A toroidal halo field is also included as prescribed in Sun \& Reich (2010), having a typical value of $2 \mu \mathrm{G}$. We include the regular field in order to make our model compatible with current information, but in fact since it is much less than the random field, this is not critical to our study.

From Sect. 2, $v_{\mathrm{c}}=(E / 1 \mathrm{GeV})^{2}\left(B_{\mathrm{ran}} / 7.5 \mu \mathrm{G}\right) \times 240 \mathrm{MHz}$, so that the full range of synchrotron frequencies used, $22 \mathrm{MHz}$ to $94 \mathrm{MHz}$, traces electron energies from roughly 0.5 to $20 \mathrm{GeV}$ for our adopted B-field.

At $408 \mathrm{MHz}$ and above, secondary leptons (above a few $\mathrm{GeV}$ ) become less important for synchrotron and the relevant leptons are measured directly without much solar modulation, while at higher frequencies $(>1 \mathrm{GHz})$ free-free emission can start to enter, so this is the best frequency to determine the random B-field. Our model for $B_{\text {ran }}$ is therefore based on fits to the data at $408 \mathrm{MHz}$. The random field is modelled as a double exponential in $(R, z)$, the free parameters being the two scale lengths (30 kpc in $R$ and $4 \mathrm{kpc}$ in $z$ ) and the local B-field: $B_{\text {ran }}=7.5 \mu \mathrm{G}^{6}$. This model reproduces the longitude and latitude distribution of synchroton at $408 \mathrm{MHz}$ sufficiently well for our purpose. Note that since we are mainly concerned with spectral shape in this paper, the absolute value of the B-field and its spatial distribution are not critical, and affect mainly the relation of electron energy to synchrotron frequency.

\section{Cosmic-ray model}

We use the GALPROP models described in Strong et al. (2010), to which we refer for details. These models have been adjusted so that the propagation parameters are consistent with CR nuclei

\footnotetext{
5 In this paper we use the temperature spectral index, as is commonly used in radioastronomy (e.g. literature in Appendix A), which is equal to to the intensity spectral index plus 2 .

6 We note that Sun et al. (2008) use an electron spectrum a factor 3 higher than the Fermi-LAT measurements, and hence obtain a random field lower than ours $(3 \mu \mathrm{G})$.
}

secondary-to-primary ratios. Only the electron injection spectrum and the B-field are varied with respect to these models; these do not affect the validity of the propagation parameters.

Electrons and positrons lose energy by synchrotron radiation, and this is included in GALPROP self-consistently using the $B_{\text {tot }}$ of the adopted model. Energy loss by inverse Compton scattering, ionization, Coulomb and bremsstrahlung are also included, although the latter three processes are of minor importance at the electron energies of interest here (see Strong \& Moskalenko 1998, for a plot of the loss processes).

We concentrate on the spectrum of electrons as determined by synchrotron data, combining these with direct measurements. Electron spectra are modelled to be consistent with Fermi-LAT data in the range $7 \mathrm{GeV}$ to $1 \mathrm{TeV}$, including an estimate of solar modulation which still has some effect at these energies. Below $7 \mathrm{GeV}$ the primary electron injection spectrum is modelled with 2 breaks. The minimal constraint is that the propagated spectrum must not be below the directly-measured spectrum (i.e. at least some solar modulation is present). Secondary electrons and positrons (from pp, p-He, He-p and He-He interactions via pion-decay) are included using the standard GALPROP treatment with locally-measured proton and Helium CR spectra as a normalization. Note that since Fermi-LAT measures electrons plus positrons, our primary electrons source effectively includes the high-energy positrons primary component measured by PAMELA (Adriani et al. 2009), so the latter is not explicitly modelled here (see e.g. Grasso et al. 2011; di Bernardo et al. 2011, for such models).

All the GALPROP parameter files for the models are available at the CDS. The plots will be made available in numerical form on request.

\section{Radio data}

\subsection{Review of information on spectral indices}

A review of radio continuum surveys and their calibration is given in Reich \& Reich (2009). We are interested in both spectral and spatial properties of the synchrotron sky, but not in the fine angular details. For the spatial distribution the most useful is the full-sky $408 \mathrm{MHz}$ survey (Haslam et al. 1982), since it has full sky coverage, a well-established calibration and zero level, and is a standard in this context. It has also the advantage that contributions from non-synchrotron components (e.g. free-free emission) are rather small. For spectral information we have assembled a set of surveys which are described in Sect. 4.2.

There are also many detailed observational studies aimed at measuring accurate sky temperatures and spectral indices at particular wavelengths, for particular sky regions. These are very valuable in providing absolute values, especially at high Galactic latitudes. They also address the question of the contribution from extragalactic radio sources. We list some representative results from the literature on spectral indices in Appendix A. In summary, there is a wealth of measurements showing that the spectral index of the synchrotron emission increases steadily from about 2.5 to 3.0 over the frequency range from tens of $\mathrm{MHz}$ to tens of GHz. While there is considerable scatter in the actual values, they still provide an essential observational constraint on any model for the CR electron + positron spectrum. Here we use a representative sample to compare with our models, but the comparison is just indicative of the general trend since the experiments cover many different sky areas. 


\subsection{Radio surveys used}

We use surveys at frequencies from $22 \mathrm{MHz}$ to $23 \mathrm{GHz}$ (and up to $94 \mathrm{GHz}$ for WMAP) to compare directly the synchrotron spectrum with the models. The surveys used are summarized below. Some were obtained directly from their authors, others from the Bonn $^{7}$ and LAMBDA ${ }^{8}$ websites. The combined zero level and extragalactic/CMB corrections were taken from the literature as stated below.

$22 \mathrm{MHz}$ : DRAO Northern hemisphere survey: Roger et al. (1999). 45 MHz: North: Maeda et al. (1999), South: Alvarez et al. (1997), combined all sky: Guzmán et al. (2011). This is complete apart from two regions of $10^{\circ}$ and $20^{\circ}$ radius out of the plane. An offset of $550 \mathrm{~K}$ was subtracted (Guzmán et al. 2011). $150 \mathrm{MHz}$ : Parkes-Jodrell Bank all sky survey: Landecker \& Wielebinski (1970). 408 MHz: Bonn-Jodrell Bank-Parkes all sky survey: Haslam et al. (1982). An offset of $3.7 \mathrm{~K}$ was subtracted (Reich \& Reich 1988a) ${ }^{9}$. 1420 MHz: Stockert-Villa Eliza all sky survey. North: Reich (1982); Reich \& Reich (1986), South: Reich et al. (2001). An offset of $2.8 \mathrm{~K}$ was subtracted (Reich et al. 2004). 2326 MHz: Rhodes southern hemisphere survey: Jonas et al. (1998). This was not used here due to restricted coverage, but will be used when the analysis is extended to lower Galactic latitudes in future. 23-94 GHz: for WMAP we used the spectral-index maps generated on the basis of WMAP polarized data by Miville-Deschênes et al. (2008), which we used to scale the $408 \mathrm{MHz}$ map to the WMAP frequencies ${ }^{10}$; in this way the synchrotron radiation is extracted essentially uncontaminated by thermal and spinning dust ${ }^{11}$.

The surveys used have full-sky (or almost full-sky) coverage apart from $22 \mathrm{MHz}$, but here the coverage is still almost complete in the sky region used. The coverage of these surveys can be seen graphically in de Oliveira-Costa et al. $(2008)^{12}$.

In order to avoid absorption effects at low frequency, and free-free emission at higher frequencies, and to avoid effects of zero-level corrections and local emission, this analysis is restricted to regions out of the Galactic plane but avoiding the polar regions, specifically $10^{\circ}<|b|<45^{\circ}$. The thermal

\footnotetext{
7 http://www.mpifr-bonn.mpg.de/survey.html

${ }^{8}$ http://lambda.gsfc.nasa.gov

9 Reich et al. (2004) give 2.7 K, Guzmán et al. (2011) give $1.6 \mathrm{~K}$, but the difference is not significant here.

${ }^{10}$ Miville-Deschênes et al. (2008) used the $408 \mathrm{MHz}$ maps uncorrected for zero offset (Miville-Deschênes, priv. comm.), so we use the same maps for consistency when applying their index maps.

11 Their re-analysis of the WMAP synchrotron data including spinning dust correction resulted in a lower intensity than previous analysis. Spinning dust emission is produced by small grains rotating and produces unpolarized radio emission. They analyzed the combination of the WMAP polarization and intensity data finding strong evidence for the presence of unpolarized spinning dust emission in the $20-60 \mathrm{GHz}$ range. They performed an analysis of the WMAP synchrotron emission at $23 \mathrm{GHz}$ where the signal to noise ratio is the highest and the polarised emission is only synchrotron. Their estimates of the intensity at this frequency are based on extrapolation of the Haslam $408 \mathrm{MHz}$ data with a spatially varying spectral index constrained by the WMAP $23 \mathrm{GHz}$ polarization data. Hence, supposing that the synchrotron spectral index does not vary with frequency over the WMAP range, they found an anomalous emission with a spectrum from 23 to $61 \mathrm{GHz}$ in accordance with the models of spinning dust.

12 They give an extensive list of radio surveys including older ones. They also provide some of them in HEALPix and use a subset for a principal components analysis with the aim of predicting the radio sky at any frequency. However issues of calibration and zero-level are not addressed by them so we do not use their results here.
}

contribution even at $1420 \mathrm{MHz}$ is then small (Reich \& Reich 1988b; Broadbent et al. 1989), a recent estimate being $15 \%$ (Dickinson et al. 2003), and less at lower frequencies (while at WMAP frequencies our maps separate out the non-thermal component as explained above). We also avoid the North Polar Spur by avoiding the region $340^{\circ}<l<40^{\circ}$, although this hardly affects the result over such large sky regions, as we have verified.

It is worth noting that lower frequency data are available (e.g. down to $1.3 \mathrm{MHz}$ from the RAE2 satellite, Novaco \& Brown 1978), discussed in Strong \& Wolfendale (1978), but these are strongly affected by absorption so are not used here.

For the spectral comparisons, the surveys and models predictions were converted to HEALPix (Górski et al. 2005) and averaged over the stated sky region. Since HEALPix has equal solid angle pixels the correct averaging is ensured.

\section{Electron and positron data}

The CR electron and positron data used are as follows: AMS01 (AMS Collaboration et al. 2002), CAPRICE94 (Boezio et al. 2000), HEAT (DuVernois et al. 2001), SANRIKU (Kobayashi 1999), BETS (Torii et al. 2001), PPT-BETS (Yoshida et al. 2008), ATIC-1-2 (Chang et al. 2008), H.E.S.S. (Aharonian et al. 2008, 2009), Fermi-LAT (Abdo et al. 2009b; Ackermann et al. 2010), PAMELA (Adriani et al. 2011). The data are taken from the CR database described in Strong \& Moskalenko (2009) ${ }^{13}$.

Fermi-LAT is taken as definitive above $7 \mathrm{GeV}$, while the extension to lower energies is based on AMS01, CAPRICE94 and HEAT. The low-energy data were taken around solar minimum with corresponding modulation levels. For HEAT the modulation levels quoted were 755 MV for 1994 and 670 MV for 1995 (DuVernois et al. 2001). The CAPRICE flight was in 1994, and quoted $600 \mathrm{MV}$ (Boezio et al. 2000). The AMS01 flight was in 1998 at the end of a solar minimum, and quoted $650 \pm 40 \mathrm{MV}$ (AMS Collaboration et al. 2002) ${ }^{14}$. The Fermi-LAT data was taken in 2008-2010 during an extreme solar minimum of polarity opposite to that of 1994-5, but for which no reliable modulation level is available. The PAMELA data were taken during 2006-2010, and a modulation of $600 \mathrm{MV}$ is used in their paper.

Fermi-LAT measured the electron spectrum from $7 \mathrm{GeV}$ to $1 \mathrm{TeV}$, with unprecedented accuracy. Above $20 \mathrm{GeV}$ the FermiLAT electron spectrum can be fitted with a simple power law with spectral index 3.04. H.E.S.S. measured the electron spectrum from $340 \mathrm{GeV}$ to $5 \mathrm{TeV}$ with a tendency to be higher than Fermi/LAT measurements in the region of overlap but in agreement within the systematics, and with a steepening above $1 \mathrm{TeV}$. ATIC-1-2 measured the spectrum from $20 \mathrm{GeV}$ to $2 \mathrm{TeV}$ and is consistent with Fermi measurements up to $300 \mathrm{GeV}$, but found a peak between 300 and $700 \mathrm{GeV}$, which is not confirmed by Fermi/LAT. Energies above $100 \mathrm{GeV}$ are not important for synchrotron (the corresponding frequencies lie beyond $1 \mathrm{THz}$ ) but are included in the model and data for completeness.

The electron spectrum $(1-625 \mathrm{GeV})$ recently measured by PAMELA (Adriani et al. 2011) is generally consistent with that measured by Fermi-LAT, although it is about $20 \%$ higher in the $7-20 \mathrm{GeV}$ range of energy overlap. The overall spectrum is also slightly softer than found by Fermi-LAT (and consistent with a rising positron fraction, see discussion in Adriani et al. 2011). We choose here to baseline on Fermi-LAT, while bearing

\footnotetext{
13 Available from http://wwW.mpe.mpg.de/ aws/propagate. html

14 The modulation levels quoted by the authors implicitly assume an interstellar CR spectrum, so the derivation may be a circular argument.
} 
in mind this difference, which does not affect our conclusions. We do not address the high-energy positron excess reported by the PAMELA group Adriani et al. (2009), since the main excess lies above the energies of importance for synchrotron, and in addition because the absolute PAMELA positron spectrum is not yet available.

Lower energy (100 MeV and below) electron data are available from the Voyager (Webber \& Higbie 2008) and Ulysses (Heber et al. 2005) spacecraft, but they are not constrained by synchrotron and will not be used here.

\section{Results}

\subsection{Pure diffusion model}

We consider first a "pure diffusion" model, with a halo height of $4 \mathrm{kpc}$. The complete set of GALPROP parameters are given in Strong et al. (2010), model z04LMPDS. The electron injection spectrum breaks at $4 \mathrm{GeV}$ and $50 \mathrm{GeV}$, with indices 1.6/2.5/2.2. The break at $50 \mathrm{GeV}$ is to fit the Fermi-LAT low-energy upturn, the break at $4 \mathrm{GeV}$ to fit low-frequency synchrotron. A cutoff at $2 \mathrm{TeV}$ is introduced to reproduce the H.E.S.S. data, although this has no effect for the synchrotron, the corresponding frequencies being far too high. Figure 1 shows the interstellar electron and positron spectra for this model, and also for various modulation levels using the force-field approximation. Figure 2 shows that this model gives a reasonable fit to the synchrotron spectrum, and illustrates the role played by the secondary leptons which have a steeper spectrum than primaries and contribute significantly to the low-frequency synchrotron. Figure 3 shows the spectral index as a function of frequency for this model, for primary and secondary leptons and for total leptons.

We next show the effect of varying the low-energy $(<4 \mathrm{GeV})$ electron injection index, from 1.0 to 2.5. Figure 4 shows the interstellar electron spectra for these models, and also for various modulation levels using the force-field approximation. It is clear that the electron data alone cannot distinguish the models due to the modulation uncertainty, so that the synchrotron constraints are essential.

Figure 5 shows that a low-energy primary electron injection index of 2.0 is at the limit of the low-frequency synchrotron data. The best fit is actually for an injection index around 1.3. Figure 6 shows the synchrotron spectral index for these models, compared to values from the literature described in Sect. 4.1.

In Fig. 7, the primary electron spectrum has been cut off below $4 \mathrm{GeV}$ to illustrate the contribution from those energies; since removing these low-energy electrons eliminates most of the low-frequency synchrotron from primaries, it shows that low-frequencies (below $100 \mathrm{MHz}$ ) are dominated by leptons with energies less than $4 \mathrm{GeV}$. Secondary leptons produce one third of the observed low-frequency intensity and hence make determination of the primary spectrum more difficult. Secondary leptons together with primaries above $4 \mathrm{GeV}$ already account for $50 \%$ of the low-frequency synchrotron.

\subsection{Reacceleration model}

We now consider a reaccleration model, also with halo height $4 \mathrm{kpc}$. The complete set of GALPROP parameters are given in Strong et al. (2010), model z04LMS; the injection spectral index above $4 \mathrm{GeV}$ has been reduced from 2.42 in that model to 2.3 to better fit the Fermi electron data above $20 \mathrm{GeV}$. The range 7-20 GeV shows a slight steepening in Fermi-LAT and PAMELA data, but no attempt has been made to reproduce this
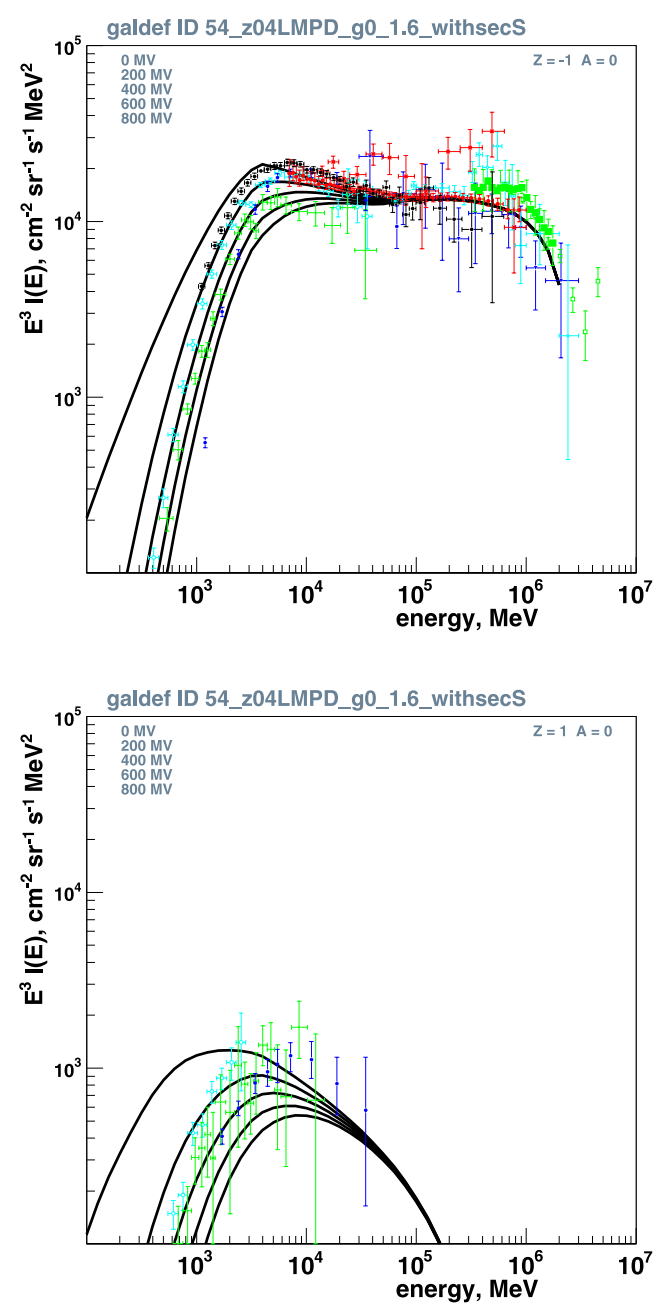

Fig. 1. Electron (upper) and positron (lower) spectra for pure diffusion model with primary low-energy electron injection index 1.6. Modulation $\Phi=0,200,400,600,800$ MV. NB Fermi-LAT includes positrons. Cyan open circles: AMS01; green crosses and filled circles: CAPRICE; blue squares: HEAT; red filled circles: FermiLAT; black filled circles: PAMELA; blue triangles: SANRIKU; red crosses: BETS, PPT-BETS; cyan open circles: ATIC-1-2; green filled and open squares: H.E.S.S. For references see text.

in the reaccelation model since we want to test an existing published model; it has no effect on our conclusions. As in the case of the pure diffusion model a cutoff above $2 \mathrm{TeV}$ has been introduced to fit the H.E.S.S. data, although this has no significance for synchrotron. The lepton and synchrotron spectra for this model are shown in Figs. 8, 9 and the synchrotron indices in Fig. 10.

It is clear that this particular reacceleration model is not consistent with the observed synchrotron spectrum, since the sum of primary and secondary leptons produces too high intensities at low frequencies, and the low-frequency spectral index is too large. It could be adjusted by making the low-energy injection index smaller, as for the pure diffusion model. However a large part of the excess comes from the secondary leptons which have a large peak due to reacceleration which makes them equal to primary electrons around $1 \mathrm{GeV}$, and which cannot be adjusted very much in this model; this peak is not present in the pure diffusion model (see comparison for secondary leptons in Fig. 2). Decreasing the B-field can improve the low-frequency fit but then the high-frequencies are under-predicted since the 

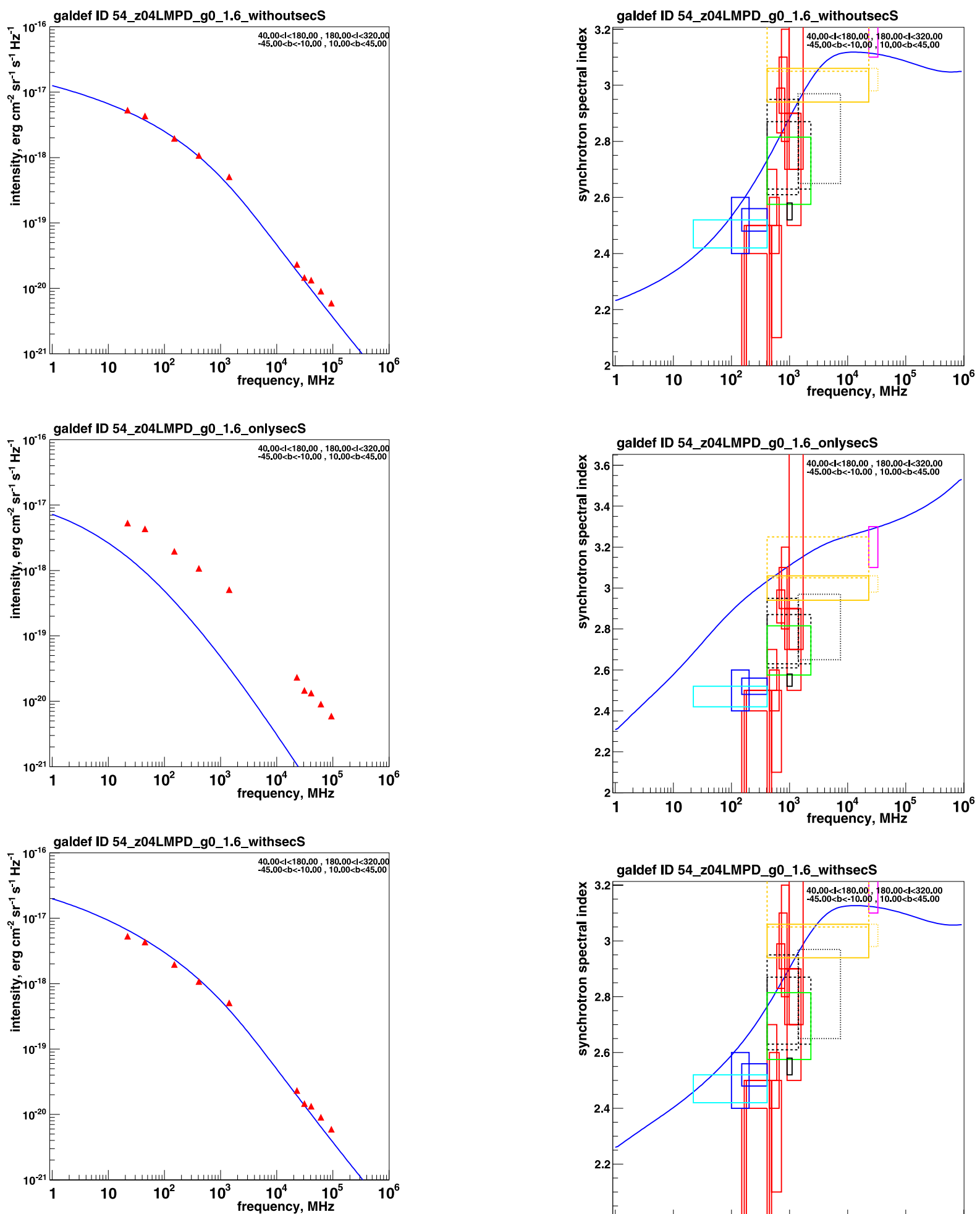

Fig. 2. Synchrotron spectra for pure diffusion model with primary lowenergy electron injection index 1.6. Synchrotron from primary electrons (upper), secondary leptons (middle) and total (lower). For synchrotron data references see Sect. 4.2.

overall spectrum is steeper than the model predicts. Only if the secondaries are removed does the synchrotron give a good fit, while the secondary production is certainly present. Arguing differently, we note that the secondaries already produce the lowenergy synchrotron intensities, which would preclude the existence of electron primaries. Either way the model is problematic for synchrotron. This does not mean that reacceleration models

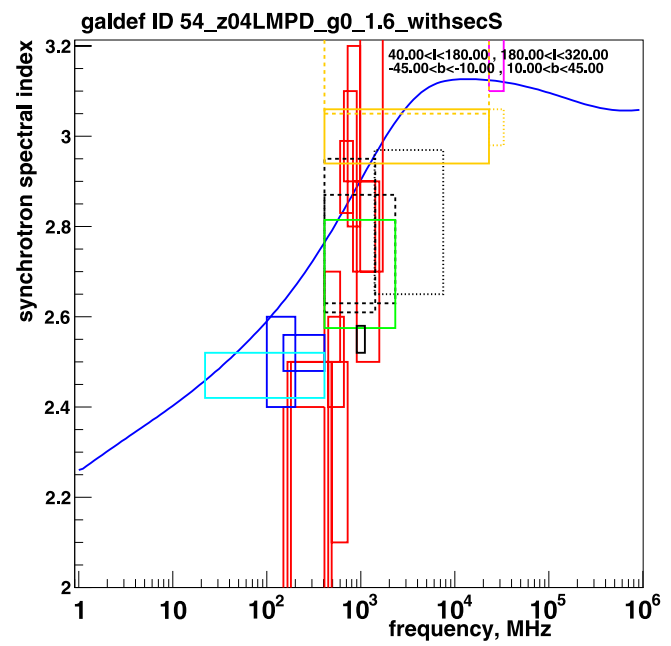

Fig. 3. Synchrotron spectral index for pure diffusion model with primary low-energy electron injection index 1.6. Synchrotron from primary electrons (upper), secondary leptons (middle) and total (lower). The experimental ranges are based on values from the literature as reviewed in the text, and are only intended to be indicative of the general trend since the measurements cover different sky areas. Data: red: Tartari et al. (2008); blue: Rogers \& Bowman (2008); cyan: Roger et al. (1999); black dashed: Giardino et al. (2002); black dotted: Platania et al. (1998); green: Platania et al. (2003); cyan: Kogut et al. (2007); orange full: Miville-Deschênes et al. (2008); orange dashed: Gold et al. (2009); orange dotted: Dunkley et al. (2009); black full: Kogut et al. (2011). 

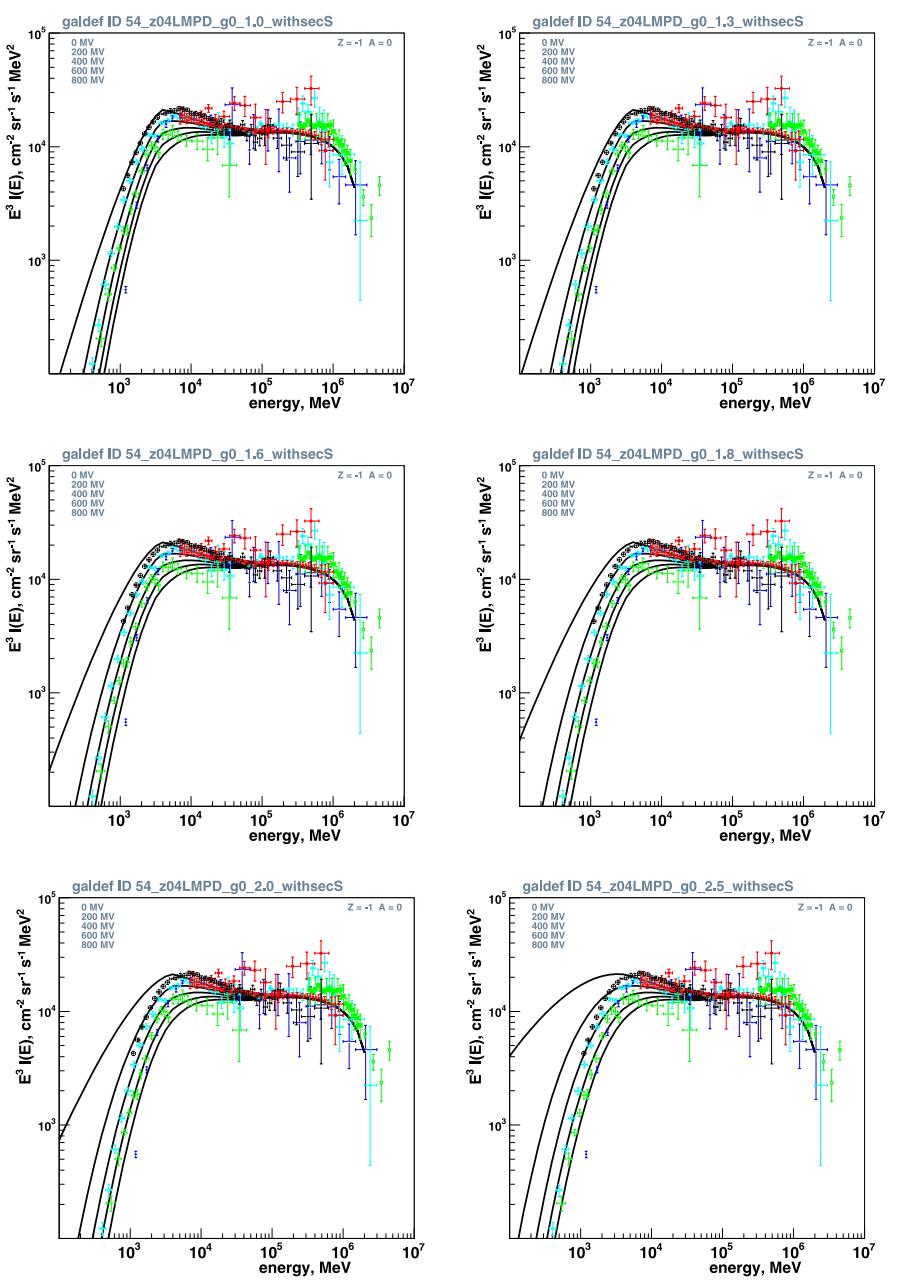

Fig. 4. Electron spectra for pure diffusion model, low-energy electron injection index 1.0, 1.3, 1.6, 1.8, 2.0, 2.5. Modulation $\Phi=0,200,400$, 600, 800 MV. Data as in Fig. 1.

are excluded by this study, but it does pose a challenge for future work on such models. Reacceleration is surely present at some level on physical grounds, but probably less than currently adopted on the basis of $\mathrm{B} / \mathrm{C}$ data.

This is an example where solar modulation can be invoked to get agreement of a model with directly-measured CR data (increasing the interstellar spectrum and modulation appropriately), but not for synchrotron which probes the interstellar spectrum.

\subsection{Low-energy spectral index from propagation?}

The low-energy injection indices deduced for the standard propagation models are unexpected (see Discussion), so it is valid to ask whether they could be produced by propagation using a more "normal" injection spectrum with index 2 . One way to do this is to reduce the energy losses by making the propagation region smaller - which means reducing the halo size. Then the steepening by propagation is reduced. This is at the expense of no more fitting $\mathrm{B} / \mathrm{C},{ }^{10} \mathrm{Be} /{ }^{9} \mathrm{Be}$ and local electron measurements, but it is worthwhile illustrating this explicitly. Figure 11 shows a model with halo height $1 \mathrm{kpc}$, but otherwise the same as the previous pure diffusion model (with its low-energy injection index 2 and halo height $4 \mathrm{kpc}$ ). (The diffusion coefficient has not been changed to fit $\mathrm{B} / \mathrm{C}$ since this would
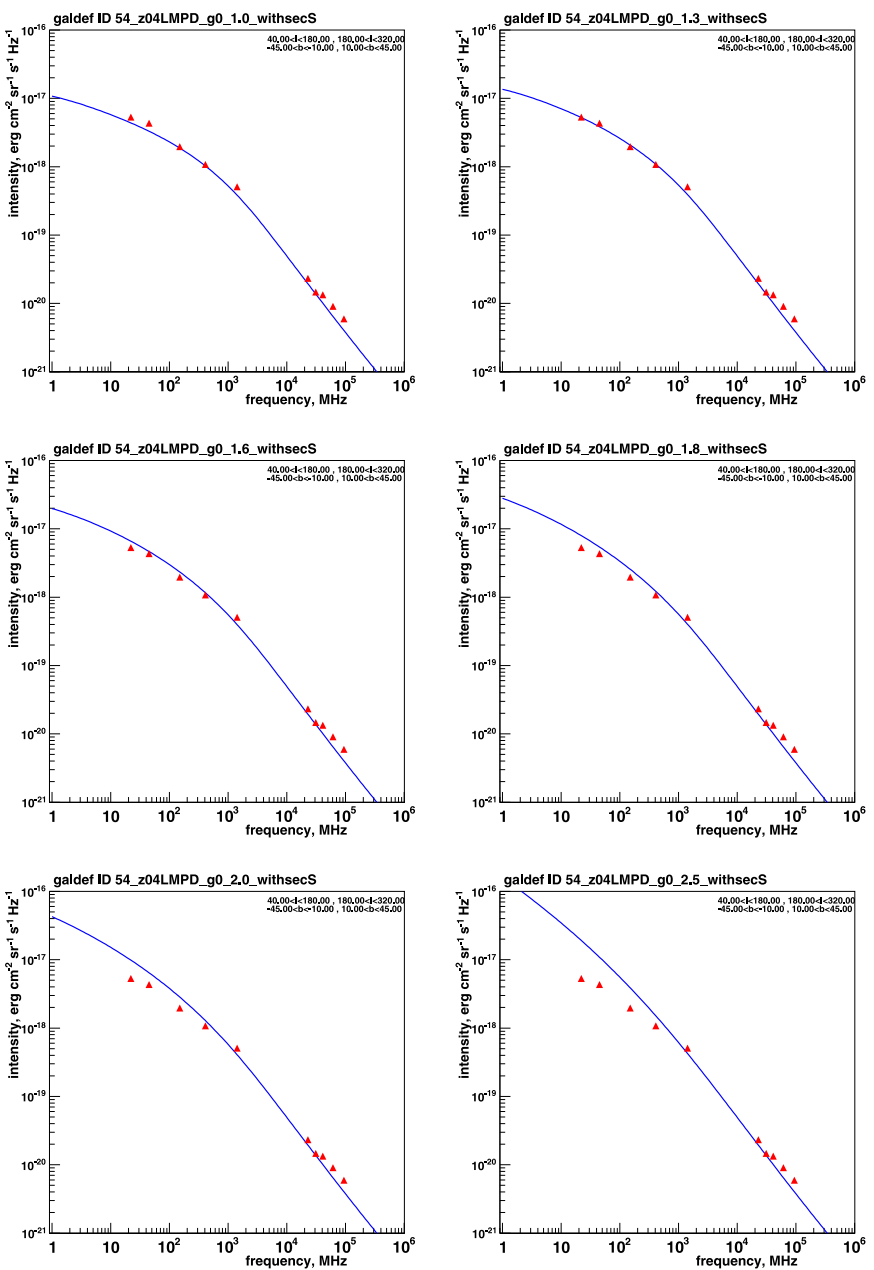

Fig. 5. Synchrotron spectra for pure diffusion model with low-energy electron injection index (left to right, top to bottom) 1.0, 1.3, 1.6, 1.8, 2.0, 2.5. Including secondary leptons. Data as in Fig. 2.

increase the propagation time and the energy losses would be unchanged from the larger halo case, and the synchrotron spectrum would be the same as before.) To get the correct synchrotron intensity, the electron flux has to be increased by 3 compared to that observed locally to compensate the smaller integration length (increasing B would just lead back to the same losses again). With this unnatural scenario we can indeed reproduce the observed synchrotron spectrum (Fig. 11). However since several other constraints are thereby violated (B/C: model too low, ${ }^{10} \mathrm{Be} /{ }^{9} \mathrm{Be}$ : model too high ${ }^{15}$, local electron spectrum: model too high) this shows the difficulty of constructing such a model consistently. There are of course other possibilities to approach this issue, but we restrict ourselves to this example here.

Another alternative to obtain the electron spectrum by propagation is to invoke an upturn in $D(E)$ at low energies, instead of the constant used normally. Figure 12 shows a model with $D(E) \propto E^{-0.5}$ for $E<4 \mathrm{GeV}$, but otherwise the same as the pure diffusion model with low-energy injection index 2 and halo height $4 \mathrm{kpc}$. It reproduces the synchrotron data, and does not require the high electron spectrum of the model with small halo height. However it will under-predict the B/C data at low energies - but this depends strongly on solar modulation and hence is not so critical. It is a possibly a more plausible scenario than the previous one since it violates less constraints. The required $D(E)$

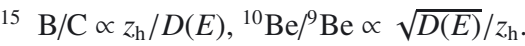



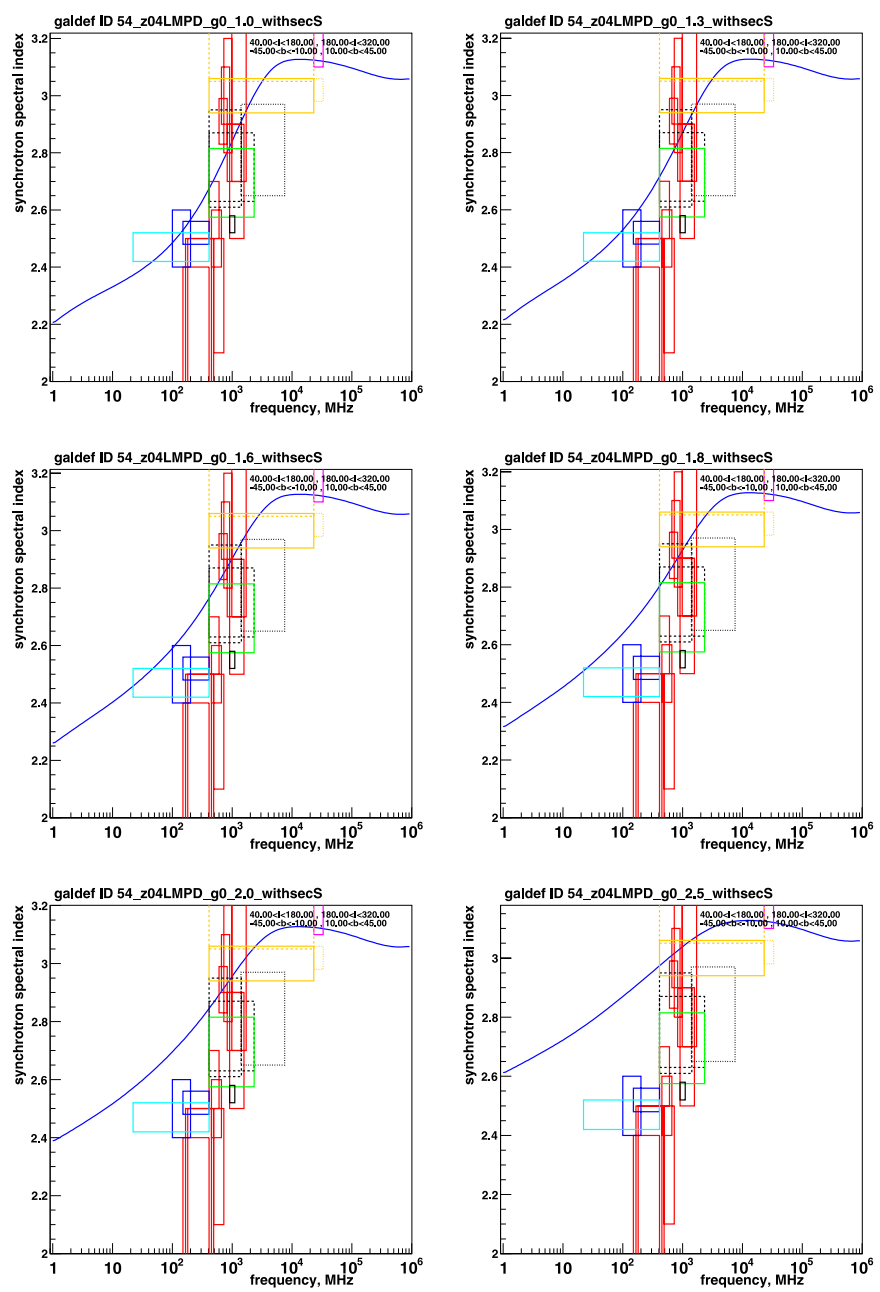

Fig. 6. Synchrotron spectral index for pure diffusion model with lowenergy electron injection index (left to right, top to bottom) 1.0, 1.3, $1.6,1.8,2.0,2.5$. Including secondary leptons. Experimental ranges are based on the references reviewed in Sect. 4.1, and are intended to be representative not exhaustive. Data as in Fig. 3.

is in fact similar to that in the wave-damping model of Ptuskin et al. (2006) and hence has also a plausible physical basis.

Other variations on the propagation could affect the electron spectrum, for example spatial variations in the diffusion coefficient, anisotropic diffusion and convection, which have not been considered here. They will not however affect our general conclusion of the need for a significant break in the electron injection spectrum. This is because any model must be constrained by the CR nuclei secondary/primary data, and the resulting modified propagation parameters will finally lead to a path-length distribution similar to our basic model, and hence similar electron energy losses.

Although we might expect a dependence of $D(E)$ on the B-field, in these models the variation of total B-field is very small (see Sect. 2.3), so that including such a dependence would have no sigificant effect. However in future such a dependence on B and its topology could be included to make the models more physically realistic.

High-energy electrons are expected to have spatial variations due to their rapid energy losses combined with the stochastic nature of the sources in space and time. A study of this effect (Strong \& Moskalenko 2001) shows that significant variations start above $10 \mathrm{GeV}$, and become large only above $100 \mathrm{GeV}$, and
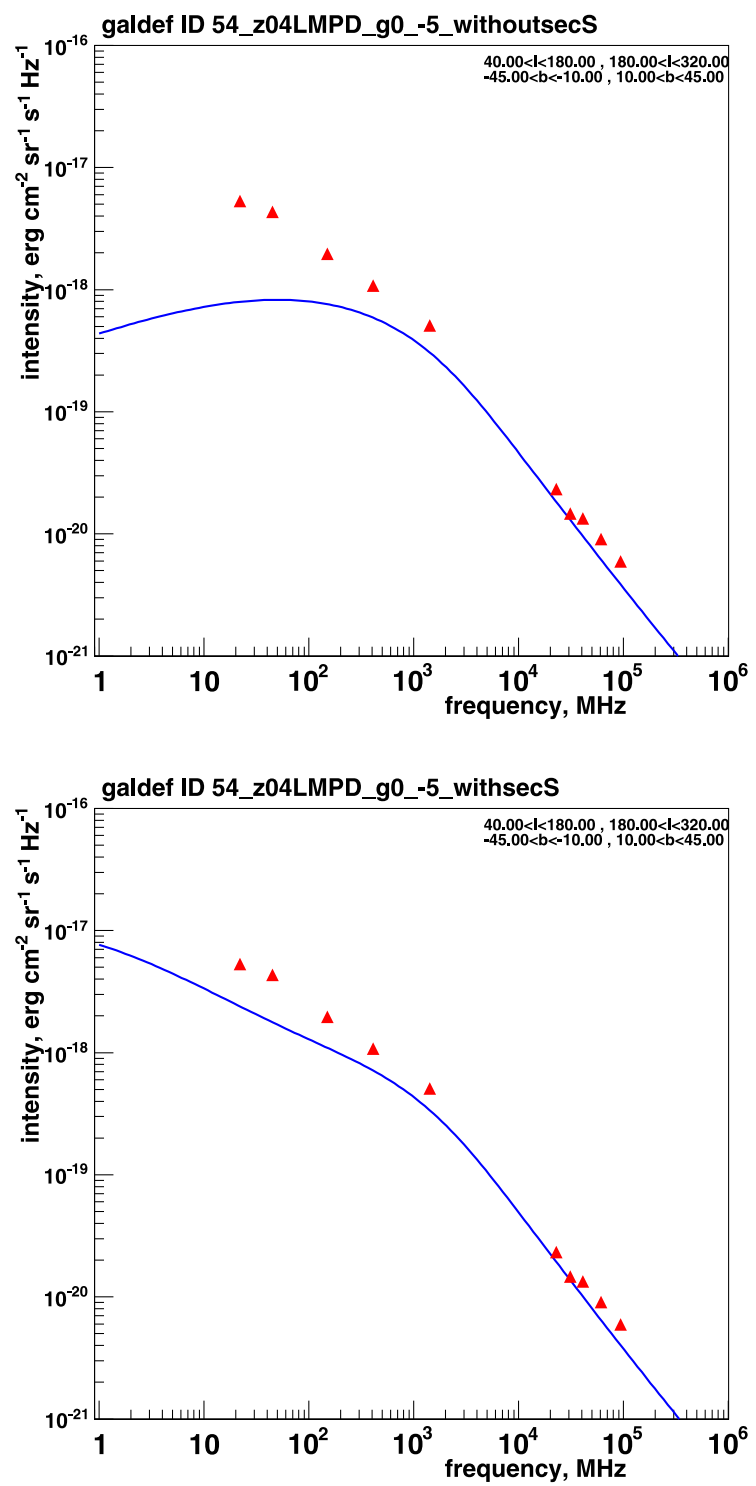

Fig. 7. Synchrotron spectra for pure diffusion model with sharp cutoff in primary electrons below $4 \mathrm{GeV}$; primary electrons only (upper), primary and secondary leptons (bottom). The contribution from secondary leptons is shown in Fig. 1. Data as in Fig. 2.

hence will not affect the low-energy synchrotron from lower energies which is the main focus of this work. In any case the synchrotron spectra we use here are integrations over large sky areas and long lines-of-sight, which will smooth out any variations and hence not affect our conclusions.

\section{Discussion}

Independent of the propagation model, the primary electron spectrum must turn over below a few $\mathrm{GeV}$, with an ambient index around 2. In the pure diffusion model this implies an injection index 1.3-1.6. It cannot cutoff completely since secondary leptons only contribute about one third of the low-frequency synchrotron, and electrons below $1 \mathrm{GeV}$ are anyway directly observed by spacecraft in the heliosphere.

While the synchrotron spectra may be subject to zero-level and scale errors, and the effects of absorption and free-free emission may affect the comparison with models, the determination of the spectral index has been performed in a robust way by 
A. W. Strong et al.: Cosmic-ray electron spectrum from synchrotron
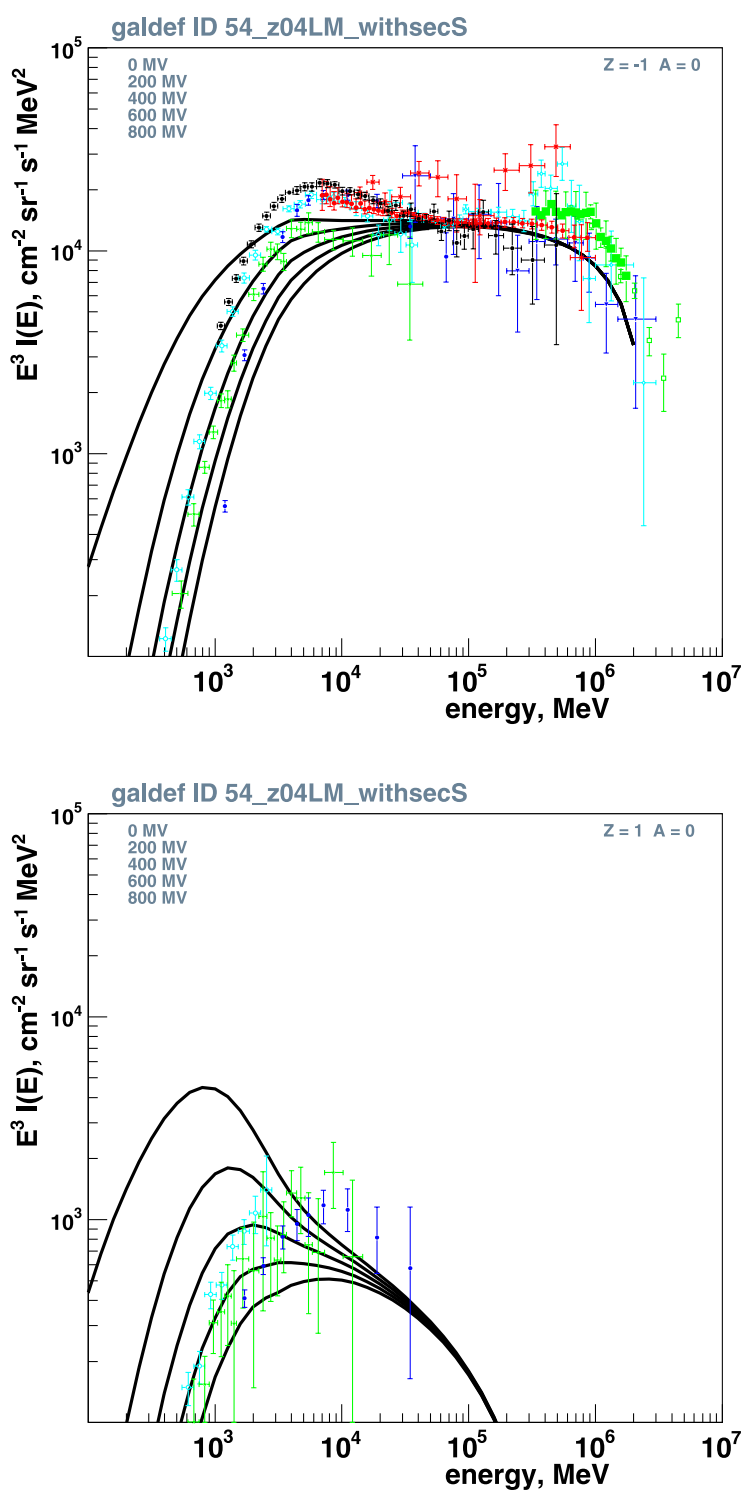

Fig. 8. Lepton spectra for diffusive reacceration model with primary low-energy electron injection index 1.6. Primary electrons (upper), secondary positrons (lower). Modulation $\Phi=0,200,400,600,800 \mathrm{MV}$. Data as in Fig. 1.

many authors and this gives a rather tight constraint on the ambient electron spectrum. The most recent determination of the spectral index 45-408 MHz (Guzmán et al. 2011) gives 2.5-2.6, implying an ambient electron index ${ }^{16}$ of 2.0-2.2 for electrons below a few $\mathrm{GeV}$. This completely excludes a continuation of the ambient electron index 3.0-3.2 measured by Fermi-LAT $>7 \mathrm{GeV}$ ) to energies below a few $\mathrm{GeV}$. Since in this range the diffusion coefficient is constant in the pure diffusion model, only energy losses steepen the spectrum slightly since escape dominates. This therefore implies an electron injection index of $<2$, consistent with the detailed analysis presented here. While the latter is a very simplified argument it supports the conclusions of the detailed calculations in a robust model-independent way. At the same time the synchrotron index $>1 \mathrm{GHz}$ has been found by many authors to be near 3 , fully consistent with the measured ambient electron spectrum above a few GeV (ambient

\footnotetext{
16 The synchrotron spectral index for an ambient electron spectrum with power-law index $p$ is approximately $\beta=2+(p-1) / 2$.
}
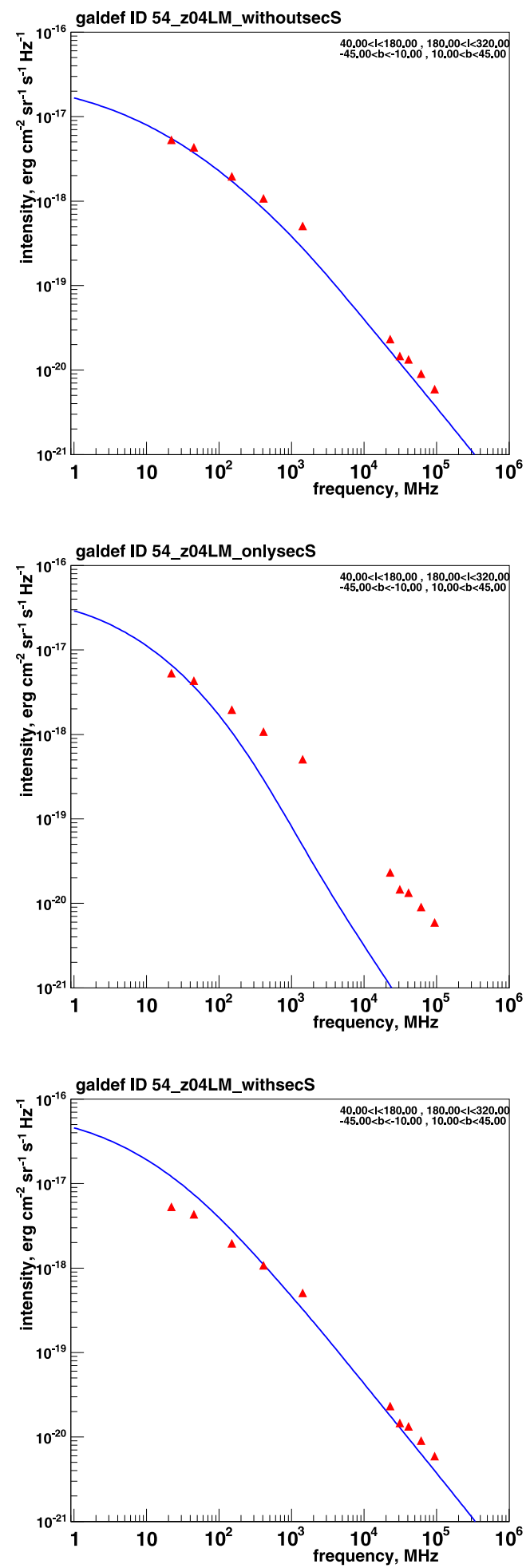

Fig. 9. Synchrotron spectra for diffusive reacceration model with primary low-energy electron injection index 1.6. Synchrotron from primary electrons (upper), secondary leptons (middle) and total (lower). Data as in Fig. 2.

index 3.0-3.2 giving synchrotron index 3.0-3.1), and an injection index 2.2 steepened by 0.5 from synchrotron/IC losses and by 0.5 due to $D(E)$.

To be more precise on this point, we can use an analytical approximation to the propagated electron spectrum for a plane parallel source distribution with diffusion (no reacceleration) 

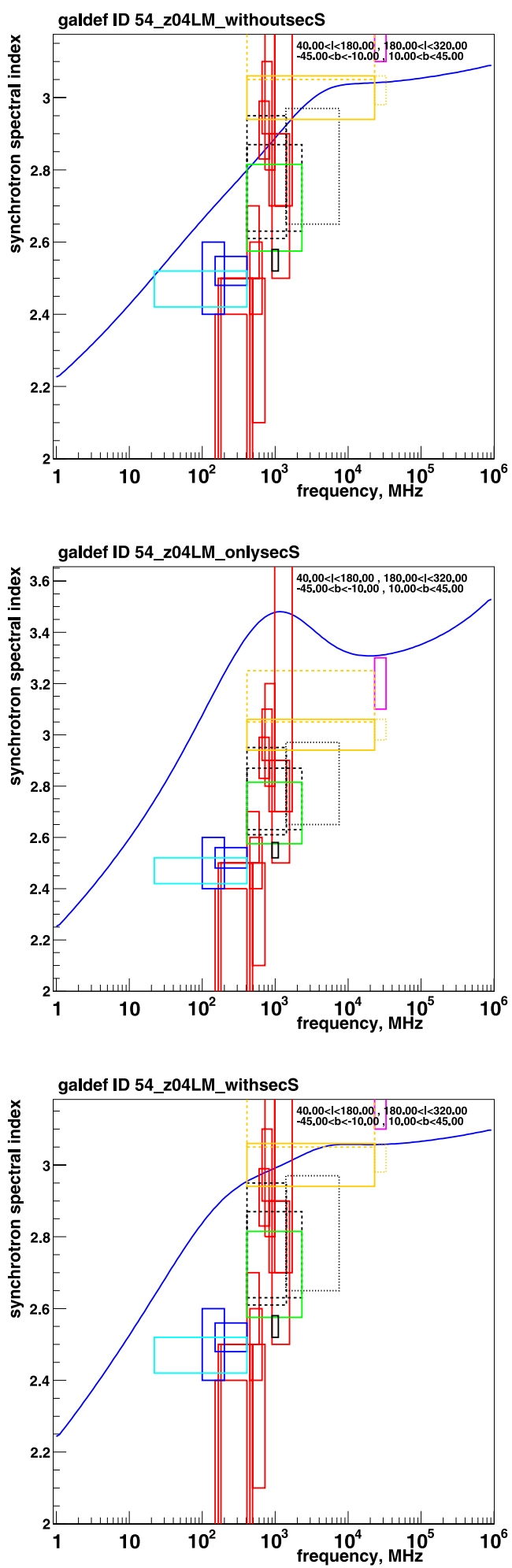

Fig. 10. Synchrotron spectral index for diffusive reacceration model with primary low-energy electron injection index 1.6. Synchrotron from primary electrons (upper), secondary leptons (middle) and total (lower). Data as in Fig. 3.

and energy losses given e.g. by Bulanov \& Dogel (1974) and Delahaye et al. (2010). The spectrum steepens by $(\delta+\alpha-1) / 2$ where $\mathrm{d} E / \mathrm{d} t \propto E^{\alpha}$ and $D(E) \propto E^{\delta}$. For synchrotron losses $\alpha=2$, so at high energies where $\delta=0.5$ the steepening is 0.75 , in accordance with our injection spectrum 2.2 and ambient spectrum 3 , and at low energies $\delta=0$ the steepening is 0.50 , consistent with
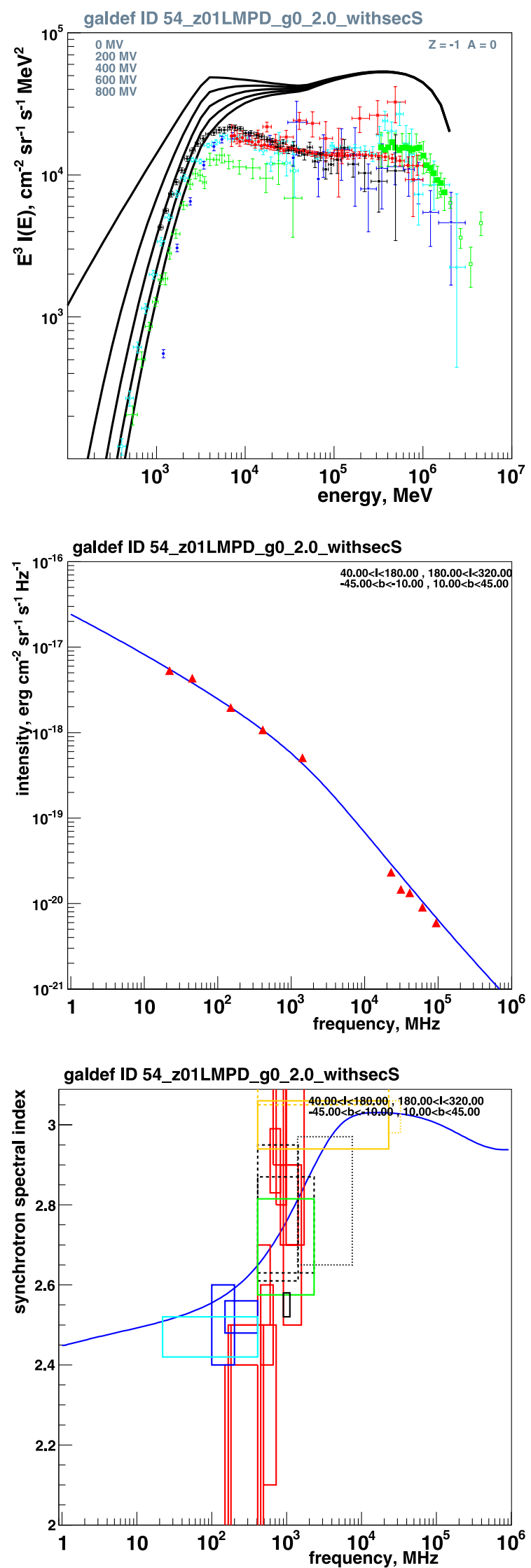

Fig. 11. Top: electron spectrum for pure diffusion model with halo height $1 \mathrm{kpc}$, low-energy electron injection index 2.0. Modulation $\Phi=$ 0, 200, 400, 600, 800 MV. Data as in Fig. 1. Centre and bottom: corresponding synchrotron spectrum and spectral index, plotted as in Fig. 5, with which this figure should be compared. 

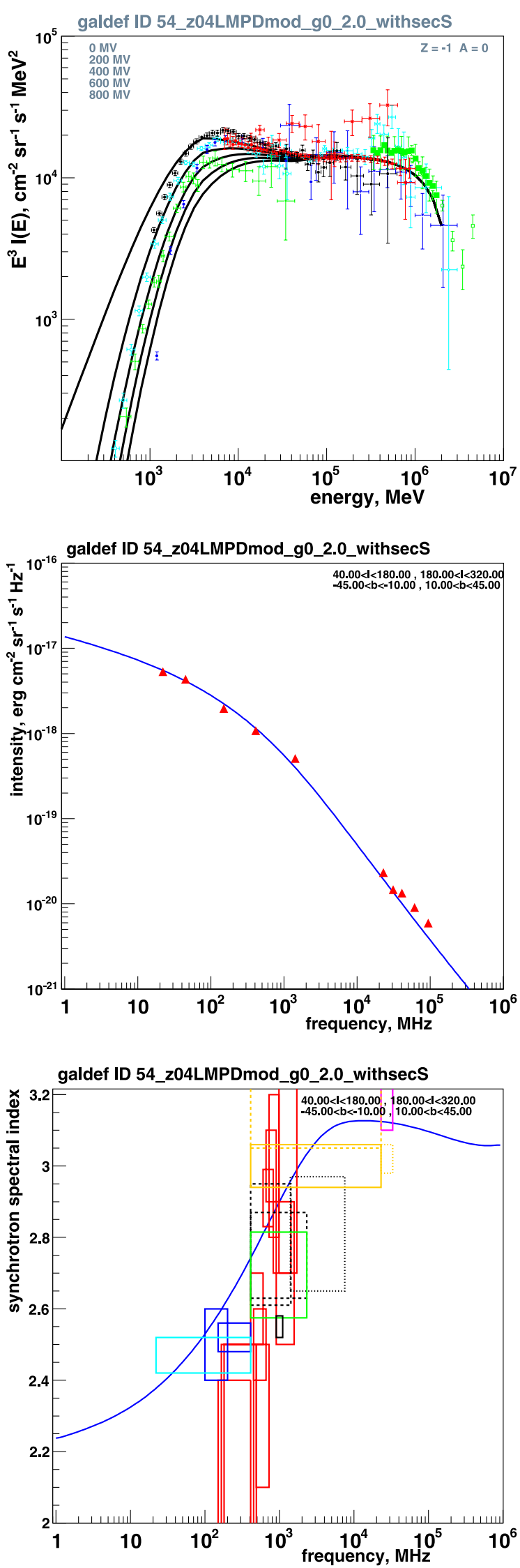

Fig. 12. Top: electron spectrum for pure diffusion model with lowenergy $D(E) \propto E^{-0.5}$, halo height $4 \mathrm{kpc}$, low-energy electron injection index 2.0. Modulation $\Phi=0,200,400,600,800$ MV. Data as in Fig. 1. Centre and bottom: corresponding synchrotron spectrum and spectral index, plotted as in Fig. 5, with which this figure should be compared. our injection index 1.3-1.6 and ambient spectrum 2 . In reality the relations are more complex due to spatial dependence etc., but the analytical form reproduces the general behaviour for synchrotron/IC-type losses, and shows it does not depend critically on the detailed GALPROP modelling.

Again we note that the determination of the ambient electron spectrum is independent of the way in which this spectrum was produced via injection and propagation. We use a particular type of parameterized model to generate physically plausible spectra, but other models would lead to the same ambient spectrum since the final criterion is consistency with synchrotron and direct measurements.

The low-energy interstellar spectrum has consequences for solar modulation: it must have a smaller effect than predicted by the force-field approximation for $\Phi=600-700 \mathrm{MV}$ generally used for such solar-mininum data (see Sect. 5); using our synchrotron-based spectrum, $\Phi \leq 200 \mathrm{MV}$ gives a better fit to data $<2 \mathrm{GeV}$. The new PAMELA data reinforces this conclusion. Since the force-field approximation is anyway known to be unreliable, and the values used are often based on an assumed interstellar spectrum, we do not pursue this further, but simply propose that the interstellar spectrum determined in this paper be used in future physical modelling of modulation. From our analysis we just suggest that the low-energy falloff in the directly measured electrons, normally attributed mainly to modulation, may instead reflect more the interstellar spectrum. We note that although synchrotron probes CR leptons but not nuclei, the improved understanding of modulation would be relevant to all species.

It is intentionally beyond the scope of this paper to speculate on the origin of the electron injection spectrum; we simply present it as an observational result posing a challenge for cosmic-ray source models. However some obvious remarks are in order. The low-energy injection spectrum is less than found in SNR; the distribution of SNR radio spectral indices Delahaye et al. (2010) is very broad - index 2.2 to 2.8 , with mean 2.48 giving an electron index 1.4 to 2.6 , with mean 1.96 . They quote an SNR electron index $2.0 \pm 0.3$. However this is for electrons inside the SNRs: the escaping flux may be harder if higher-energy electrons escape more easily as might be expected. At high energies our derived injection index 2.2 suggests a steepening relative to the spectrum inside the remnants, but at lower energies this may not hold. A recent study of escape of electrons from SNR is given by Ohira et al. (2011), and Ohira et al. (2010), which includes among many effects an escape time decreasing with energy, and hence a hardening of the escape spectrum. Our results will constrain such models, which predict a complex escape spectrum.

Other sources of electrons may also contribute, for example pulsar wind nebula (PWN) have radio indices 2-2.3, (Reynolds et al. 2011) giving electron index 1-1.6, similar to our lowenergy injection index. While PWN clearly cannot produce the high-energy spectrum, it is possible that they play a role at low energies. Pulsars produce electrons with index 1.5-1.9 (with a cutoff at several GeV) (Grasso et al. 2011) again similar to our low-energy index. The attempt to construct a model with various source types to reproduce the data is beyond the scope of the present work, but in fact it is not easy to imagine how hard spectrum low-energy sources plus steeper spectrum high-energy sources could be combined to produce the observed composite spectrum.

Finally we compare the electron injection spectrum with that of nuclei, in particular protons. In the GALPROP models used here, the nuclei injection spectrum has index, above/below a 
break energy of $9 \mathrm{GeV} /$ nucleon, of $1.8 / 2.25$ for the plain diffusion model, and 1.98/2.42 for the reacceleration model. These values have been chosen to agree with $\mathrm{CR}$ data for the given propagation parameters derived from $\mathrm{B} / \mathrm{C}$ etc. Thus the lowenergy nuclei index is slighty larger than what we have deduced for electrons (1.3-1.6 for the plain diffusion model, 1.6 for the reacceleration model). However the nuclei are strongly affected by solar modulation at low energies, and unlike the electrons there is no equivalent of the synchrotron tracer for the interstellar spectrum (pion-decay gamma rays may be constraining but are sensitive mainly to protons above a few $\mathrm{GeV}$ ). So a nuclei injection spectrum equal to that of electrons would be acceptable, if indeed that should be predicted by a theory of CR acceleration, but is not required by the data.

\section{Conclusions}

Our main conclusion is that the interstellar CR electron spectrum must turn over rather sharply below a few GeV. This result is independent of how the spectrum is formed by injection and propagation. The low-energy falloff in the directly measured electrons, normally attributed just to modulation, may instead reflect mainly the interstellar spectrum. The (model-dependent) injection index implied for the primary electrons is 1.3-1.6 below a few $\mathrm{GeV}$, and 2.1-2.3 at higher energies. The standard reacceleration model is not consistent with the observed synchrotron spectrum, since the total from primary and secondary leptons exceeds the measured synchrotron at low frequencies. While not excluding reacceleration models, it does pose a challenge to be addressed.

We show that it is still possible to obtain the ambient electron spectrum by propagation even for a more conventional injection index of 2 , but at the expense of violating other constraints. A low-energy upturn in the diffusion coefficient is the most promising model of this kind.

Therefore combining synchrotron data with direct measurements of CR provides unique and essential constraints on the interstellar electron spectrum. These results have implications for interstellar gamma rays especially at low energies (Porter et al. 2008), and also for high energies (see Strong 2011, for a recent review). Exploiting the complementary information on cosmic rays and synchrotron gives new constraints and has implications for gamma rays. This connection is especially relevant now in view of the ongoing Planck and Fermi missions, and in future new radio astronomy instruments like LOFAR and direct measurements by AMS-02.

There are of course limits to what conclusions based on a model like GALPROP can achieve since the full complexity of the Galaxy and physical processes can never be reproduced. A key is the combination of constraints from many different data types ("multi-messenger"), but still it is hard to break the degeneracy between the source injection spectrum and propagation even with secondary/primary ratios etc. In future as CR sources are better understood via radio, X-ray and gamma-ray observations we should be able to make more basic progress on this topic.

Acknowledgements. We acknowledge useful comments from I. Moskalenko, T. Porter, G. Jóhannesson and A. Vladimirov. We thank the referee for critical comments which helped to improve this paper.

\section{Appendix A: Radio spectral index data}

Starting with lower frequencies, Roger et al. (1999) used the $22 \mathrm{MHz}$ and $408 \mathrm{MHz}$ surveys to derive average high latitude $\beta=2.47$, with a variation of about 0.05 . Rogers \& Bowman (2008) measured over a continuous band $100-200 \mathrm{MHz}, \beta=$ $2.5 \pm 0.1$; combining with other surveys $150-408 \mathrm{MHz} \beta=$ $2.52 \pm 0.04$ at high latitudes. This paper contains a useful summary table of $\beta$ back to 1962. Guzmán et al. (2011) use the recent $45 \mathrm{MHz}$ all-sky map together with the $408 \mathrm{MHz}$ Haslam map to derive $\beta=2.5-2.6$ over most of the sky for these frequencies.

At higher frequencies, Reich \& Reich (1988b), separating thermal and nonthermal components for $408-1420 \mathrm{MHz}$ found a non-thermal index $\beta=2.85-3.1$. Reich et al. (2004) give spectral indices for 45-408-1420-22800 MHz, and give a table of zero-level corrections. Spectral index maps. $\beta(408-1420 \mathrm{MHz})=2.6-2.7$ away from plane and loops. Giardino et al. (2002) find 408-1420 MHz $\beta=2.78 \pm 0.17$ and 408-2326 MHz $\beta=2.75 \pm 0.12$. Platania et al. (2003) from a full-sky analysis of 408, 1420 and $2326 \mathrm{MHz}$ survey find $\beta=2.695$ with a dispersion of 0.12. Platania et al. (1998) using radiometers at a high-altitude site at 1400-7500 MHz: $\beta=2.81 \pm 0.16$. Zannoni et al. (2008) report TRIS absolute measurements at $0.6,0.82,2.5 \mathrm{GHz}$, drift scans at $\delta-42^{\circ}$ (this is Paper I, II = Gervasi et al. 2008b, III = Tartari et al. 2008). Gervasi et al. (2008a) derive the extragalactic source contribution to the background from 151 to $8440 \mathrm{MHz}$. Tartari et al. (2008) using TRIS III find a synchrotron halo with $\beta=2.9-3.1$ 600-820 MHz. This paper discusses zero errors in other surveys (their Table 9: 150, 408, 820, $1420 \mathrm{MHz}$ surveys). They find that $\beta$ increases from 2.2 to 2.8 from $150 \mathrm{MHz}$ to $1420 \mathrm{MHz}$. They give a spectrum in two directions $\left(9 \mathrm{~h}, 42^{\circ}, 10 \mathrm{~h}, 42^{\circ}\right)$ corrected for zero level (their Fig. 7) which can serve as a standard: it shows large steepening from 150 to $1420 \mathrm{MHz}$ ). They give the variation of $\beta(600-820 \mathrm{MHz})$ along $\delta=42^{\circ}$ : it is mainly in the range 2.8 to 3.2 (their Fig. 5). This paper contains an extensive discussion of spectral indices past and present, and its relation to electron spectrum. $T_{\mathrm{gal}}=T-T_{\mathrm{off}}=T-\left(T_{\mathrm{ex}}+\right.$ $\left.T_{\text {cmb }}\right)+T_{\text {zero }}$. From their Table 9 , the correction to the $408 \mathrm{MHz}$ Haslam survey is $T_{\text {zero }}=+3.9 ; T_{\mathrm{ex}}=2.65, T_{\mathrm{cmb}}=2.82$ gives $T_{\text {off }}=1.57$ compared to Reich \& Reich (1988a, their Table 7): $T_{\text {zero }}=+2.1, T_{\text {off }}=3.7 \pm 0.85$.

Kogut et al. (2011) (ARCADE2) at 3, 8, $10 \mathrm{GHz}: \beta_{\text {sync }}=$ $2.55 \pm 0.03$ using a $408 \mathrm{MHz}$ template, but they use $\operatorname{cosec}(\mathrm{b})$ and CII correlations. Poles/coldest regions $\beta_{\text {sync }}=2.57 \pm 0.03$. These values are significantly lower than those normally found (see above: $2.7-3.1)^{17}$.

Turning to the highest frequencies, Hinshaw et al. (2007) using WMAP 3-year data found $\beta=3.15-3.5$ for $23-61 \mathrm{GHz}$. Dunkley et al. (2009) used WMAP 5-year polarized maps to derive synchrotron $\beta=3.02 \pm 0.04$ (at WMAP frequencies). They show skymaps of $\beta$, and find no latitude dependence unlike Kogut et al. (2007) (WMAP 3-year data) who found an increase from 3.05 to 3.25 from the Galactic plane to the poles. Gold et al. (2009) used WMAP 5-year data to derive

\footnotetext{
17 In a related ARCADE2 study, Fixsen et al. (2011) claim a 3-90 GHz extragalactic background about a factor 6 higher than expected from radio sources at $1 \mathrm{GHz}$. Excess claimed to have $\beta=2.60$ from $22 \mathrm{MHz}$ to $10 \mathrm{GHz}$. To model the Galactic emission they use a cosec(b) analysis, which would not be sensitive to a large halo (see their discussion, correlation with CII). This was followed up in Seiffert et al. (2011). who attribute the excess to underestimated Galactic emission or unaccounted radio sources, or some combination of both. Vernstrom et al. (2011) use radio source counts to show that the claimed extragalactic background is hard to explain as the sum of sources to the currently observed flux limits, and that a extra population at lower fluxes would be required. See also Singal et al. (2010), who also relate the radio to the extragalactic $\mathrm{X}$ - and gamma-ray backgrounds.
} 
synchrotron $\beta=3.15 \pm 0.10$ (roughly from their Fig. 16), including polarized-only analysis. The latitude profile shows a lower index for $|b|<10^{\circ}: 2.8$, (unlike Dunkley et al. 2009, who found no latitude variation on the same 5-year data; but like Kogut et al. 2007, who found similar variation slightly different values) on the 3-year WMAP data. Miville-Deschênes et al. (2008) give maps of $23 \mathrm{GHz}$ intensity and $\beta 408 \mathrm{MHz}-23 \mathrm{GHz}$ using WMAP polarized emission maps, especially useful for comparing with synchrotron models since it selects out the (polarized) synchrotron emission in a model-independent way. Testori et al. (2008) give spectral index distributions using polarized intensity for the $1.435 \mathrm{GHz}$ survey and $22 \mathrm{GHz}$ WMAP. It peaks at $\beta=2.7-3.0$, but ranges from 1.8 to 3.6 due to depolarization in the plane and being near the noise level. Reich et al. (2004) discuss zero-level errors in WMAP, and conclude, using T-T plots, that $\beta(1420 \mathrm{MHz}-22.8 \mathrm{GHz})$ decreases from 3.1 to 2.8 after correction, nearer to the $408-1420 \mathrm{MHz}$ value.

\section{References}

Abdo, A. A., Ackermann, M., Ajello, M., et al. 2009a, Phys. Rev. Lett., 103, 251101

Abdo, A. A., Ackermann, M., Ajello, M., et al. 2009b, Phys. Rev. Lett., 102, 181101

Ackermann, M., Ajello, M., Atwood, W. B., et al. 2010, Phys. Rev. D, 82, 092004 Adriani, O., Barbarino, G. C., Bazilevskaya, G. A., et al. 2009, Nature, 458, 607 Adriani, O., Barbarino, G. C., Bazilevskaya, G. A., et al. 2011, Phys. Rev. Lett., 106, 201101

Aharonian, F., Akhperjanian, A. G., Barres de Almeida, U., et al. 2008, Phys. Rev. Lett., 101, 261104

Aharonian, F., Akhperjanian, A. G., Anton, G., et al. 2009, A\&A, 508, 561

Alvarez, H., Aparici, J., May, J., \& Olmos, F. 1997, A\&AS, 124, 315

AMS Collaboration, Aguilar, M., Alcaraz, J., Allaby, J., et al. 2002, Phys. Rep., 366,331

Boezio, M., Carlson, P., Francke, T., et al. 2000, ApJ, 532, 653

Bringmann, T., Donato, F., \& Lineros, R. A. 2011, [arXiv: 1106.4821]

Broadbent, A., Osborne, J. L., \& Haslam, C. G. T. 1989, MNRAS, 237, 381

Bulanov, S. V., \& Dogel, V. A. 1974, Ap\&SS, 29, 305

Chang, J., Adams, J. H., Ahn, H. S., et al. 2008, Nature, 456, 362

de Oliveira-Costa, A., Tegmark, M., Gaensler, B. M., et al. 2008, MNRAS, 388, 247

Delahaye, T., Lavalle, J., Lineros, R., Donato, F., \& Fornengo, N. 2010, A\&A, 524, A51

di Bernardo, G., Evoli, C., Gaggero, D., et al. 2011, Astropart. Phys., 34, 528

Dickinson, C., Davies, R. D., \& Davis, R. J. 2003, MNRAS, 341, 369

Dunkley, J., Spergel, D. N., Komatsu, E., et al. 2009, ApJ, 701, 1804

DuVernois, M. A., Barwick, S. W., Beatty, J. J., et al. 2001, ApJ, 559, 296

Fixsen, D. J., Kogut, A., Levin, S., et al. 2011, ApJ, 734, 5

Gervasi, M., Tartari, A., Zannoni, M., Boella, G., \& Sironi, G. 2008a, ApJ, 682, 223

Gervasi, M., Zannoni, M., Tartari, A., Boella, G., \& Sironi, G. 2008b, ApJ, 688, 24

Ghisellini, G., Guilbert, P. W., \& Svensson, R. 1988, ApJ, 334, L5

Giardino, G., Banday, A. J., Górski, K. M., et al. 2002, A\&A, 387, 82

Gold, B., Bennett, C. L., Hill, R. S., et al. 2009, ApJS, 180, 265

Górski, K. M., Hivon, E., Banday, A. J., et al. 2005, ApJ, 622, 759

Grasso, D., Profumo, S., Strong, A. W., et al. 2011, Nucl. Instrum. Meth. Phys. Res. A, 630, 48

Guzmán, A. E., May, J., Alvarez, H., \& Maeda, K. 2011, A\&A, 525, A138

Haslam, C. G. T., Salter, C. J., Stoffel, H., \& Wilson, W. E. 1982, A\&AS, 47, 1

Heber, B., Kopp, A., Fichtner, H., \& Ferreira, S. E. S. 2005, Adv. Space Res., 35,605
Hinshaw, G., Nolta, M. R., Bennett, C. L., et al. 2007, ApJS, 170, 288

Jaffe, T. R., Banday, A. J., Leahy, J. P., Leach, S., \& Strong, A. W. 2011, MNRAS, 416, 1152

Jonas, J. L., Baart, E. E., \& Nicolson, G. D. 1998, MNRAS, 297, 977

Kobayashi, T. 1999, in International Cosmic Ray Conference, 3, 61

Kogut, A., Dunkley, J., Bennett, C. L., et al. 2007, ApJ, 665, 355

Kogut, A., Fixsen, D. J., Levin, S. M., et al. 2011, ApJ, 734, 4

Landecker, T. L., \& Wielebinski, R. 1970, Aust. J. Phys. Astrophys. Suppl., 16, 1

Longair, M. S. 2010, High Energy Astrophysics

Maeda, K., Alvarez, H., Aparici, J., May, J., \& Reich, P. 1999, A\&AS, 140, 145

Miville-Deschênes, M., Ysard, N., Lavabre, A., et al. 2008, A\&A, 490, 1093

Novaco, J. C., \& Brown, L. W. 1978, ApJ, 221, 114

Ohira, Y., Murase, K., \& Yamazaki, R. 2010, A\&A, 513, A17

Ohira, Y., Yamazaki, R., Kawanaka, N., \& Ioka, K. 2011, ApJ, submitted [arXiv: 1106.1810$]$

Orlando, E., Strong, A. W., Moskalenko, I. V., et al. 2009, International Cosmic Ray Conference, Lodz [arXiv: 0907. 0553]

Platania, P., Bensadoun, M., Bersanelli, M., et al. 1998, ApJ, 505, 473

Platania, P., Burigana, C., Maino, D., et al. 2003, A\&A, 410, 847

Porter, T. A., Moskalenko, I. V., Strong, A. W., Orlando, E., \& Bouchet, L. 2008, ApJ, 682, 400

Ptuskin, V. S., Moskalenko, I. V., Jones, F. C., Strong, A. W., \& Zirakashvili, V. N. 2006, ApJ, 642, 902

Reich, W. 1982, A\&AS, 48, 219

Reich, P., \& Reich, W. 1986, A\&AS, 63, 205

Reich, P., \& Reich, W. 1988a, A\&AS, 74, 7

Reich, P., \& Reich, W. 1988b, A\&A, 196, 211

Reich, W., \& Reich, P. 2009, in IAU Symp., 259, 603

Reich, P., Testori, J. C., \& Reich, W. 2001, A\&A, 376, 861

Reich, P., Reich, W., \& Testori, J. C. 2004, in The Magnetized Interstellar Medium, ed. B. Uyaniker, W. Reich, \& R. Wielebinski, 63

Reynolds, S. P., Gaensler, B. M., \& Bocchino, F. 2011, Space Sci. Rev., 46

Roger, R. S., Costain, C. H., Landecker, T. L., \& Swerdlyk, C. M. 1999, A\&AS, 137,7

Rogers, A. E. E., \& Bowman, J. D. 2008, AJ, 136, 641

Seiffert, M., Fixsen, D. J., Kogut, A., et al. 2011, ApJ, 734, 6

Singal, J., Stawarz, Ł., Lawrence, A., \& Petrosian, V. 2010, MNRAS, 409, 1172

Strong, A. W. 2011, Proceedings of the ICATPP Conference on Cosmic Rays for Particle and Astroparticle Physics, Villa Olmo, Como, Italy, 7-8 October, 2010 (World Scientific) [arXiv: 1101.1381]

Strong, A. W., \& Moskalenko, I. V. 1998, ApJ, 509, 212

Strong, A. W. \& Moskalenko, I. V. 2001, Proc. 27th International Cosmic Ray Conference, Hamburg, 5, 1964

Strong, A. W., \& Moskalenko, I. V. 2009, 31st International Cosmic Ray Conference, Lodz [arXiv:0907.0565]

Strong, A. W., \& Wolfendale, A. W. 1978, J. Phys. G Nucl. Phys., 4, 1793

Strong, A. W., Moskalenko, I. V., \& Reimer, O. 2000, ApJ, 537, 763

Strong, A. W., Moskalenko, I. V., \& Reimer, O. 2004a, ApJ, 613, 962

Strong, A. W., Moskalenko, I. V., Reimer, O., Digel, S., \& Diehl, R. 2004b, A\&A, 422, L47

Strong, A. W., Moskalenko, I. V., \& Ptuskin, V. S. 2007, Ann. Rev. Nucl. Part. Sci., 57, 285

Strong, A. W., Porter, T. A., Digel, S. W., et al. 2010, ApJ, 722, L58

Sun, X., \& Reich, W. 2010, Res. Astron. Astrophys., 10, 1287

Sun, X. H., Reich, W., Waelkens, A., \& Enßlin, T. A. 2008, A\&A, 477, 573

Tartari, A., Zannoni, M., Gervasi, M., Boella, G., \& Sironi, G. 2008, ApJ, 688, 32

Testori, J. C., Reich, P., \& Reich, W. 2008, A\&A, 484, 733

Torii, S., Tamura, T., Tateyama, N., et al. 2001, ApJ, 559, 973

Trotta, R., Jóhannesson, G., Moskalenko, I. V., et al. 2011, ApJ, 729, 106

Vernstrom, T., Scott, D., \& Wall, J. 2011, MNRAS, 415, 3641

Webber, W. R., \& Higbie, P. R. 2008, J. Geophys. Res., Space Phys., 113, A11106

Yoshida, K., Torii, S., Yamagami, T., et al. 2008, Adv. Space Res., 42, 1670

Zannoni, M., Tartari, A., Gervasi, M., et al. 2008, ApJ, 688, 12 